\title{
Colistin Resistance among Enterobacteriaceae Isolated from Clinical Samples in Gaza Strip
}

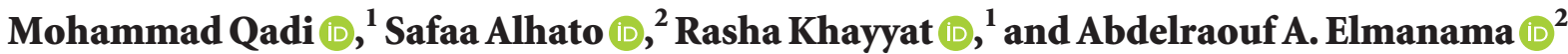 \\ ${ }^{1}$ Department of Biomedical Sciences, Faculty of Medicine and Health Sciences, An-Najah National University, P.O.Box. 7, \\ Nablus, Palestine, State of Palestine \\ ${ }^{2}$ Department of Medical Laboratory Sciences Faculty of Health Sciences Islamic University of Gaza, Gaza Strip, Gaza, \\ Palestine, State of Palestine \\ Correspondence should be addressed to Mohammad Qadi; m.qadi@najah.edu
}

Received 9 December 2020; Accepted 13 April 2021; Published 20 April 2021

Academic Editor: Louis Detolla

Copyright ( 2021 Mohammad Qadi et al. This is an open access article distributed under the Creative Commons Attribution License, which permits unrestricted use, distribution, and reproduction in any medium, provided the original work is properly cited.

\begin{abstract}
Bacterial infections, especially drug-resistant infections, are a major global health issue. The emergence of multidrug-resistant (MDR) strains of Enterobacteriaceae and the lack of new antibiotics have worrisome prospects for all of humanity. Colistin is considered the last-line drug for MDR Gram-negative bacteria (GNB), and it is often used for treatment of respiratory infections caused by MDR-GNB. In recent years, there has been a marked increase in the incidence of colistin-resistant infections. The main objective of this study was to investigate the presence of colistin resistance among clinical GNB isolated from Gaza Strip hospitals. Clinical Enterobacteriaceae isolates (100) were obtained from microbiology laboratories of the hospitals of different geographical locations in Gaza Strip Governorate over a period of six months. Samples were cultured, and bacterial identification was performed by standard microbiological procedures. Enterobacteriaceae isolates were tested for their antimicrobial susceptibility by the disk diffusion method and the MIC method for colistin. Varying degrees of susceptibility were observed for the isolates against the tested antimicrobials even within members of the same antimicrobial class. Amikacin was the most effective drug (74\%), followed by chloramphenicol (48\%), fosfomycin, and gentamicin (45\%). High resistance was recorded against trimethoprim (85\%) and tetracycline (83\%). Only 59\% of the tested isolates were interpreted as susceptible, while $41 \%$ was classified as resistant. The highest resistance to colistin was found to be among the Proteus spp. (63.2\%), followed by Serratia spp. (57.1\%). The lowest resistance was observed among Klebsiella isolates (31.6\%). Only 39.0\% of meropenem-resistant Enterobacteriaceae was susceptible to colistin, while $45.8 \%$ of imipenem-resistant Enterobacteriaceae was susceptible to colistin. The overall resistance to colistin was high (41\%) among tested clinical isolates. Furthermore, $89 \%$ was MDR. These limit and complicate treatment options for the infections caused by Enterobacteriaceae in Gaza Strip. This calls for immediate actions to control and monitor the use of antimicrobials in general and colistin in particular.
\end{abstract}

\section{Introduction}

The global rise in the phenomenon of antimicrobial resistance in the fight against bacterial infections is very disturbing, and concerns regarding this issue are increasing, as it complicates infectious disease treatment and increases the financial burden on healthcare systems [1].

Bacterial infections, especially drug-resistant infections, are a major global health issue. The emergence of multidrugresistant (MDR) strains of bacteria and the lack of new antibiotics have worrisome prospects for all of humanity. A recent report suggests failing to control drug-resistant infections that may cause an excess of 10 million deaths per year and may cost up to US\$ 100 trillion by 2050 [2].

Colistin is considered the last-line drug for MDR Gramnegative bacteria (GNB) [3], and it is often used for treatment of respiratory infections caused by MDR-GNB. In recent years, there has been a marked increase in the incidence of colistin-resistant infections [4].

Colistin resistance is caused by decreases in the net negative charge of the outer membrane, loss of lipid A, or efflux pumps, and the most common resistance mechanism 
in Enterobacteriaceae is the covalent modification of the lipid A moiety of lipopolysaccharide (LPS) via cationic substitution; these modifications neutralize the negative charge of LPS and subsequently reduce the binding affinity of colistin for its target [5].

Increasing the use of colistin for treatment of infections caused by GNB has led to the emergence of colistin resistance in several countries worldwide. Although resistance to polymyxins is generally less than $10 \%$, it is higher in the Mediterranean and Southeast Asia (Korea and Singapore), where colistin resistance rates are continually increasing [6].

Antimicrobial susceptibility data for 178 carbapenemase-producing Klebsiella pneumoniae (KPC-Kp) isolates revealed that $76(43 \%)$ were resistant to colistin [7].

An unpublished report in Gaza showed high resistance percentages among Escherichia coli isolated from poultry farms. Colistin use in the poultry industry in Gaza strip is indiscriminate, and farmers use it without prescription. This may contribute to resistance to colistin among animals' bacteria which finds its way to humans through food and other means.

This study aims at investigating the resistance of Enterobacteriaceae bacteria to antimicrobials in general and particularly to colistin.

\section{Materials and Methods}

2.1. Bacterial Isolate Sources. One hundred clinical isolates (Enterobacteriaceae) were obtained from microbiology laboratories belonging to the Ministry of Health hospitals (20 isolates from each hospital: Al-Shifa, European Gaza Hospital (EGH), Al-Aqsa, Nasser, and Indonesian hospitals) during the period from December 2018 to May 2019. The isolates were presumptively identified by the microbiology laboratory of the corresponding hospitals and were reidentified at the Islamic University of Gaza microbiology laboratories.

\subsection{Inclusion and Exclusion Criteria. All Enterobacteriaceae} isolated during the study period in the mentioned hospitals were included, and no Enterobacteriaceae isolates were excluded.

2.3. Isolate Collection and Transportation. Clinical isolates were collected on a weekly basis from the five laboratories. Each lab was supplied with freshly prepared triple sugar iron agar slants (TSIA). Technicians streaked each isolate into a separate TSIA slant, incubated overnight, and placed in a refrigerator for temporary storage. Within one week of isolation, the isolates were transported to the microbiology laboratory at the Islamic university of Gaza for identity confirmation and antimicrobial testing.

2.4. Isolate Identification. After purification streak, each isolate was subjected to conventional biochemical tests such as TSIA, Simmons citrate, urease, methyl red, sulfide-indolemotility, and oxidase in addition to Gram staining.
2.5. Antimicrobial Susceptibility Testing. Each isolate was subjected to antimicrobial susceptibility testing using the disk diffusion method in accordance to the procedures and guidance of clinical laboratory sciences institute [8]. Commercial antimicrobial disks (Liofilchem, Italy) were placed onto the surface of preswabbed Muller-Hinton agar plates with a $0.5 \mathrm{McF}$ arland calibrated inoculum of the test organism. Plates were placed in a refrigerator for 15 minutes to allow proper diffusion of antimicrobials and then incubated overnight at $37^{\circ} \mathrm{C}$. Interpretation of the results was performed according to antimicrobial disk manufacturer tables.

2.6. Colistin Minimum Inhibitory Concentration (MIC). The microbroth dilution method using the 96-microtiter plate was employed. Serial dilutions of pure colistin (SigmaAldrich Inc.) were tested against a standardized bacterial inoculum. After 16-20 hours of incubation, $20 \mathrm{ul}$ of tetrazolium chloride was added to each well and incubated for 15 minutes. MIC was calculated based on colour development.

Because CLSI does not provide breakpoints for Enterobacteriaceae when testing colistin, we used the European Committee on Antimicrobial Susceptibility Testing (EUCAST) MIC breakpoints for colistin for the purpose of interpretation: $\leq 2 \mathrm{mg} / \mathrm{l}$ susceptible and $>2 \mathrm{mg} / \mathrm{l}$ resistant [9].

\section{Results}

The Enterobacteriaceae isolates used in this study were obtained from clinical samples, collected from different sources as shown in Figure 1. The pus group includes pus from different sources such as wound and ear charge samples.

The distribution of E. coli, Klebsiella spp., Proteus group, Serratia spp., and Enterobacter spp. isolates according to their source is given in Table 1 .

E. coli was isolated mainly from urine samples $(74.5 \%)$, followed by pus (21.6), while the Proteus group was isolated mainly from pus (47.4\%), followed by urine samples $(36.8 \%)$. In general, urine culture constituted the major source of the isolates (58\%), followed by pus (31\%) as given in Table 2.

3.1. Antimicrobial Resistance of Clinical Enterobacteriaceae Isolates. Varying degrees of susceptibilities were observed for the isolates against the tested antimicrobials even within members of the same antimicrobial class. Amikacin was the most effective drug with $74 \%$, followed by chloramphenicol (48\%) and fosfomycin and gentamicin (45\%). High resistance was recorded against trimethoprim (85\%) and tetracycline (83\%). 89 isolates (89\%) were MDR, as given in Table 3.

3.2. Colistin MIC. Bacterial isolates that showed resistance at a concentration higher than $2 \mathrm{mg} / \mathrm{l}$ were classified as resistant. Only $59 \%$ of the tested isolated were interpreted as 


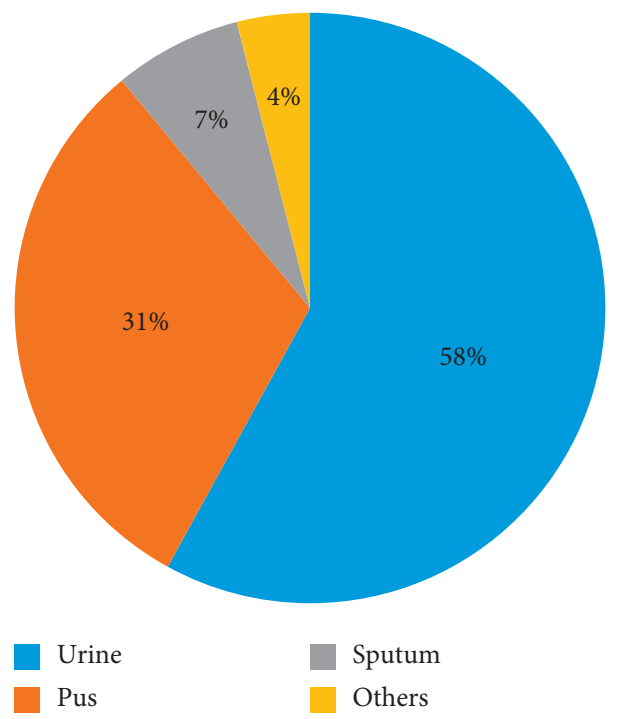

Figure 1: Isolates investigated in this study were obtained from sample groups ( $*$ Others, 2 from blood culture and 1 each from cerebrospinal fluid and high vaginal swab). The collected Enterobacteriaceae consisted of E. coli (51\%), Klebsiella spp. (19\%), Proteus group (19\%), Serratia spp. (7\%), and Enterobacter spp. (4\%).

TABle 1: Enterobacteriaceae recovered from clinical specimen distribution by hospitals.

\begin{tabular}{|c|c|c|c|c|c|c|}
\hline \multirow{2}{*}{ Enterobacteriaceae } & \multicolumn{5}{|c|}{ Hospital name } & \multirow{2}{*}{ Total } \\
\hline & Indonesian hospital & Al-Shifa hospital & Al-Aqsa hospital & Nasser medical complex & European gaza hospital & \\
\hline \multirow{2}{*}{ E. coli } & 8 & 15 & 11 & 7 & 10 & 51 \\
\hline & $15.7 \%$ & $29.4 \%$ & $21.6 \%$ & $13.7 \%$ & $19.6 \%$ & $100.0 \%$ \\
\hline \multirow{2}{*}{ Enterobacter spp. } & 2 & 0 & 1 & 0 & 1 & 4 \\
\hline & $50.0 \%$ & $0.0 \%$ & $25.0 \%$ & $0.0 \%$ & $25.0 \%$ & $100.0 \%$ \\
\hline \multirow{2}{*}{ Proteus group } & 2 & 1 & 1 & 11 & 4 & 19 \\
\hline & $10.5 \%$ & $5.3 \%$ & $5.3 \%$ & $57.9 \%$ & $21.1 \%$ & $100.0 \%$ \\
\hline \multirow{2}{*}{ Serratia spp. } & 2 & 2 & 0 & 0 & 3 & 7 \\
\hline & $28.6 \%$ & $28.6 \%$ & $0.0 \%$ & $0.0 \%$ & $42.9 \%$ & $100.0 \%$ \\
\hline \multirow{2}{*}{ Klebsiella spp. } & 6 & 2 & 7 & 2 & 2 & 19 \\
\hline & $31.6 \%$ & $10.5 \%$ & $36.8 \%$ & $10.5 \%$ & $10.5 \%$ & $100.0 \%$ \\
\hline \multirow{2}{*}{ Total } & 20 & 20 & 20 & 20 & 20 & 100 \\
\hline & $20.0 \%$ & $20.0 \%$ & $20.0 \%$ & $20.0 \%$ & $20.0 \%$ & $100.0 \%$ \\
\hline
\end{tabular}

$P$ value $=0.002$.

susceptible, while $41 \%$ was classified as resistant. Table 4 provides the MIC value obtained for 100 isolates tested against colistin sulfate.

The highest resistance to colistin was found to be among the Proteus group (63.2\%), followed by Serratia (57.1\%). The lowest resistance was observed among Klebsiella isolates (31.6\%) as given in Table 5. Despite variations in resistance among the different genera, no statistical difference was detected $(P=0.154)$.

3.3. Colistin Resistance among Carbapenem-Resistant Enterobacteriaceae. Colistin is being used as last choice for carbapenem-resistant Enterobacteriaceae, and therefore, resistance against colistin was compared to that of imipenem and meropenem. Only $39.0 \%$ of meropenem-resistant
Enterobacteriaceae was susceptible to colistin, while $45.8 \%$ of imipenem-resistant Enterobacteriaceae was susceptible to colistin as given in Table 6 .

\section{Discussion}

One hundred clinical isolates of Enterobacteriaceae were tested against 16 antimicrobials. The lowest antimicrobial resistance was for amikacin (19\%); this percent is higher than the percent reported years ago, where the percent of amikacin resistance among GNB responsible for nosocomial bacteremia was $3.9 \%$, while among community-acquired isolates, it was $1.8 \%$ [10]. On the other hand, it is lower than amikacin resistance among ESBL-producing isolates of Enterobacteriaceae in a study performed by Tayh et al. in 2019 [11] which was 33.3\% 
TABLE 2: Distribution of the isolates according to clinical sample type.

\begin{tabular}{|c|c|c|c|c|c|c|c|}
\hline \multirow{2}{*}{ Isolate } & \multicolumn{6}{|c|}{ Sample type } & \multirow{2}{*}{ Total } \\
\hline & Urine & Pus & Sputum & H.V.S & CSF & Blood & \\
\hline E. coli & $\begin{array}{c}38 \\
74.5 \%\end{array}$ & $\begin{array}{c}11 \\
21.6 \%\end{array}$ & $\begin{array}{c}1 \\
2.0 \%\end{array}$ & $\begin{array}{c}1 \\
2.0 \% \\
\end{array}$ & $\begin{array}{c}0 \\
0.0 \%\end{array}$ & $\begin{array}{c}0 \\
0.0 \%\end{array}$ & $\begin{array}{c}51 \\
100.0 \% \\
\end{array}$ \\
\hline Enterobacter spp. & $\begin{array}{c}1 \\
25.0 \% \\
\end{array}$ & $\begin{array}{c}2 \\
50.0 \% \\
\end{array}$ & $\begin{array}{c}1 \\
25.0 \% \\
\end{array}$ & $\begin{array}{c}0 \\
0.0 \% \\
\end{array}$ & $\begin{array}{c}0 \\
0.0 \% \\
\end{array}$ & $\begin{array}{c}0 \\
0.0 \% \\
\end{array}$ & $\begin{array}{c}4 \\
100.0 \% \\
\end{array}$ \\
\hline Proteus group & $\begin{array}{c}7 \\
36.8 \% \\
\end{array}$ & $\begin{array}{c}9 \\
47.4 \% \\
\end{array}$ & $\begin{array}{c}1 \\
5.3 \% \\
\end{array}$ & $\begin{array}{c}0 \\
0.0 \% \\
\end{array}$ & $\begin{array}{c}0 \\
0.0 \% \\
\end{array}$ & $\begin{array}{c}2 \\
10.5 \% \\
\end{array}$ & $\begin{array}{c}19 \\
100.0 \% \\
\end{array}$ \\
\hline Serratia spp. & $\begin{array}{c}0 \\
0.0 \% \\
\end{array}$ & $\begin{array}{c}5 \\
71.4 \% \\
\end{array}$ & $\begin{array}{c}2 \\
28.6 \% \\
\end{array}$ & $\begin{array}{c}0 \\
0.0 \% \\
\end{array}$ & $\begin{array}{c}0 \\
0.0 \% \\
\end{array}$ & $\begin{array}{c}0 \\
0.0 \% \\
\end{array}$ & $\begin{array}{c}7 \\
100.0 \% \\
\end{array}$ \\
\hline Klebsiella spp. & $\begin{array}{c}12 \\
63.2 \% \\
\end{array}$ & $\begin{array}{c}4 \\
21.1 \% \\
\end{array}$ & $\begin{array}{c}2 \\
10.5 \% \\
\end{array}$ & $\begin{array}{c}0 \\
0.0 \% \\
\end{array}$ & $\begin{array}{c}1 \\
5.3 \% \\
\end{array}$ & $\begin{array}{c}0 \\
0.0 \% \\
\end{array}$ & $\begin{array}{c}19 \\
100.0 \% \\
\end{array}$ \\
\hline Total & $\begin{array}{c}58 \\
58.0 \%\end{array}$ & $\begin{array}{c}31 \\
31.0 \%\end{array}$ & $\begin{array}{c}7 \\
7.0 \%\end{array}$ & $\begin{array}{c}1 \\
1.0 \%\end{array}$ & $\begin{array}{c}1 \\
1.0 \%\end{array}$ & $\begin{array}{c}2 \\
2.0 \%\end{array}$ & $\begin{array}{c}100 \\
100.0 \%\end{array}$ \\
\hline
\end{tabular}

$P=0.006$.

TABle 3: Percentage of antimicrobial resistance of Enterobacteriaceae tested against 15 antimicrobials.

\begin{tabular}{|c|c|c|c|}
\hline Antimicrobial & $S$ & $I$ & $R$ \\
\hline Amikacin & 74 & 7 & 19 \\
\hline Chloramphenicol & 48 & 11 & 41 \\
\hline Fosfomycin & 45 & 8 & 47 \\
\hline Gentamicin & 45 & 6 & 49 \\
\hline Ciprofloxacin & 43 & 5 & 52 \\
\hline Meropenem & 39 & 10 & 51 \\
\hline Imipenem & 33 & 22 & 45 \\
\hline Ceftriaxone & 23 & 14 & 63 \\
\hline Ceftazidime & 16 & 7 & 77 \\
\hline Trimethoprim & 14 & 1 & 85 \\
\hline Tetracycline & 13 & 4 & 83 \\
\hline Cefotaxime & 12 & 9 & 79 \\
\hline Cefuroxime & 12 & 6 & 82 \\
\hline Ampicillin & 11 & 0 & 89 \\
\hline Trimethoprim-sulfamethoxazole & 8 & 8 & 84 \\
\hline
\end{tabular}

TABLE 4: MIC values for various Enterobacteriaceae.

\begin{tabular}{|c|c|c|c|c|c|c|}
\hline MIC in $\mathrm{mg} / \mathrm{l}$ & E. coli & Enterobacter & Proteus group & Serratia & Klebsiella & Total \\
\hline 16 & $\begin{array}{c}6 \\
33.3 \%\end{array}$ & $\begin{array}{c}1 \\
5.6 \%\end{array}$ & $\begin{array}{c}8 \\
44.4 \%\end{array}$ & $\begin{array}{c}1 \\
5.6 \%\end{array}$ & $\begin{array}{c}2 \\
11.1 \%\end{array}$ & $\begin{array}{c}18 \\
100 \%\end{array}$ \\
\hline 8 & $\begin{array}{c}6 \\
40.0 \%\end{array}$ & $\begin{array}{c}1 \\
6.7 \%\end{array}$ & $\begin{array}{c}4 \\
26.7 \%\end{array}$ & $\begin{array}{c}1 \\
6.7 \%\end{array}$ & $\begin{array}{c}3 \\
20.0 \%\end{array}$ & $\begin{array}{c}15 \\
100 \%\end{array}$ \\
\hline 4 & $\begin{array}{c}5 \\
62.5 \% \\
\end{array}$ & $\begin{array}{c}0 \\
0.0 \% \\
\end{array}$ & $\begin{array}{c}0 \\
0.0 \% \\
\end{array}$ & $\begin{array}{c}2 \\
25.0 \% \\
\end{array}$ & $\begin{array}{c}1 \\
12.5 \% \\
\end{array}$ & $\begin{array}{c}8 \\
100 \% \\
\end{array}$ \\
\hline 2 & $\begin{array}{c}3 \\
50.0 \% \\
\end{array}$ & $\begin{array}{c}1 \\
16.7 \% \\
\end{array}$ & $\begin{array}{c}0 \\
0.0 \% \\
\end{array}$ & $\begin{array}{c}1 \\
16.7 \% \\
\end{array}$ & $\begin{array}{c}1 \\
16.7 \% \\
\end{array}$ & $\begin{array}{c}6 \\
100 \% \\
\end{array}$ \\
\hline 1 & $\begin{array}{c}9 \\
64.3 \%\end{array}$ & $\begin{array}{c}0 \\
0.0 \%\end{array}$ & $\begin{array}{c}3 \\
21.4 \%\end{array}$ & $\begin{array}{c}0 \\
0.0 \%\end{array}$ & $\begin{array}{c}2 \\
14.3 \%\end{array}$ & $\begin{array}{c}14 \\
100 \%\end{array}$ \\
\hline 0.5 & $\begin{array}{c}22 \\
56.4 \%\end{array}$ & $\begin{array}{c}1 \\
2.6 \%\end{array}$ & $\begin{array}{c}4 \\
10.3 \%\end{array}$ & $\begin{array}{c}2 \\
5.1 \%\end{array}$ & $\begin{array}{c}10 \\
25.6 \%\end{array}$ & $\begin{array}{c}39 \\
100 \%\end{array}$ \\
\hline Total & $\begin{array}{c}51 \\
51.0 \%\end{array}$ & $\begin{array}{c}4 \\
4.0 \%\end{array}$ & $\begin{array}{c}19 \\
19.0 \%\end{array}$ & $\begin{array}{c}7 \\
7.0 \%\end{array}$ & $\begin{array}{c}19 \\
19.0 \%\end{array}$ & $\begin{array}{c}100 \\
100 \%\end{array}$ \\
\hline
\end{tabular}

The highest antimicrobial resistance was against ampicillin (89\%); this percent is higher than the percent reported in 2003 [12], which was $71.6 \%$. This means that ampicillin resistance increases with the rise of its use over time. Another study [11] conducted on ESBL-producing Enterobacteriaceae in urinary tract infections showed that the 
Table 5: MIC values for various Enterobacteriaceae.

\begin{tabular}{|c|c|c|c|}
\hline \multirow{2}{*}{ Enterobacteriaceae } & \multicolumn{2}{|c|}{ Colistin MIC } & \multirow{2}{*}{ Total } \\
\hline & Susceptible & Resistant & \\
\hline \multirow{2}{*}{ E. coli } & 34 & 17 & 51 \\
\hline & $66.7 \%$ & $33.3 \%$ & $100 \%$ \\
\hline \multirow{2}{*}{ Enterobacter } & 2 & 2 & 4 \\
\hline & $50.0 \%$ & $50.0 \%$ & $100 \%$ \\
\hline \multirow{2}{*}{ Proteus group } & 7 & 12 & 19 \\
\hline & $36.8 \%$ & $63.2 \%$ & $100 \%$ \\
\hline \multirow{2}{*}{ Serratia } & 3 & 4 & 7 \\
\hline & $42.9 \%$ & $57.1 \%$ & $100 \%$ \\
\hline \multirow{2}{*}{ Klebsiella } & 13 & 6 & 19 \\
\hline & $68.4 \%$ & $31.6 \%$ & $100 \%$ \\
\hline \multirow{2}{*}{ Total } & 59 & 41 & 100 \\
\hline & $59.0 \%$ & $41.0 \%$ & $100 \%$ \\
\hline
\end{tabular}

$P=0.154$.

TABle 6: Colistin resistance among carbapenem-resistant Enterobacteriaceae.

\begin{tabular}{lccccccrrr}
\hline Colistin & \multicolumn{2}{c}{ Susceptible } & \multicolumn{2}{c}{ Intermediate } & \multicolumn{2}{c}{ Resistant } & Total (\%) & $P$ value \\
\hline Meropenem & & & & & & & & \\
$\quad$ Susceptible & 28 & $47.5 \%$ & 8 & $13.5 \%$ & 23 & $39.0 \%$ & 59 & 59.0 & 0.014 \\
$\quad$ Resistant & 11 & $26.8 \%$ & 2 & $4.9 \%$ & 28 & $68.3 \%$ & 41 & 41.0 \\
\hline Imipenem & & & & & & & & \\
$\quad$ Susceptible & 16 & $27.1 \%$ & 16 & $27.1 \%$ & 27 & $45.8 \%$ & 59 & 59.0 & 0.192 \\
$\quad$ Resistant & 17 & $41.5 \%$ & 6 & $14.6 \%$ & 18 & $43.9 \%$ & 41 & 41.0 \\
\hline
\end{tabular}

percent of ampicillin resistance in ESBL-producing isolates was $100 \%$, and in non-ESBL-producing isolates, it was $58.2 \%$. Moreover, in a study conducted on E.coli isolated from chicken droppings in Gaza strip [13], the percent of ampicillin resistance was $100 \%$.

Our results showed that the percent of colistin resistance was $41 \%$. Interestingly, this percent is lower than the percent reported in [14], a study which revealed a percent of $63.4 \%$ of colistin resistance and higher than the percent of colistin resistance in the study conducted on E. coli isolates [13] which was $14.5 \%$.

Percent of E. coli resistance to colistin was 33.3\%, and for Klebsiella, it was $31.6 \%$.

It is obvious that we are reporting a higher percent of colistin resistance in comparison to the published data in Kuwait in 2018 by Alfoiuzan et al. [15] where the team reported resistance of $4.3 \%$ for E. coli and $7.7 \%$ for Klebsiella.

Carbapenem-resistant Gram-negative pathogens have become a major healthcare burden in the $21^{\text {st }}$ century, and treatment options had been limited to agents such as colistin and tigecycline in combination with other antibiotics [16]. In this study, only 39\% of meropenem-resistant Enterobacteriaceae was susceptible to colistin, while $45.8 \%$ of imipenem-resistant Enterobacteriaceae was susceptible to colistin. Our findings highlight how much resistance to colistin has increased within the last ten years. For example, in England, ten years ago, the activity of colistin was evaluated against 81 carbapenem-resistant Enterobacteriaceae isolates, and colistin was active against $75 / 81$ isolates (92.6\%) [17].
In [18] a study of colistin resistance in Klebsiella pneumoniae and E.coli strains isolated from cancer patients, $45 \%$ of colistin-resistant isolates were meropenem resistant.

In [19] a study, the percent of imipenem and meropenem resistant GNB was $8.1 \%$ and $0.8 \%$ respectively, and in the [11] study, the percentage of imipenem resistance in ESBLproducing isolates of Enterobacteriaceae was 20\%. Those reported percentages are much less than the percent determined by our study, which was $43.9 \%$ for imipenem and $68.3 \%$ for meropenem. This calls for setting policies to (1) prevent misuse and overuse of antibiotics in general and carbapenems, and colistin in particular, especially with the high rate of MDR detected in our study, and (2) applying procedures for infection control and screening policies for antibiotic resistance on a routine basis.

\section{Conclusion}

In conclusion, overall resistance to colistin was high (41\%), and in the same context, MDR percentage was $89 \%$ among tested clinical isolates. These limit and complicate treatment options of infection caused by Enterobacteriaceae in Gaza Strip, which in turn calls for immediate actions to control and monitor the use of antimicrobials in general and colistin in particular.

\section{Data Availability}

The data used to support the findings of this study are included within the article. 


\section{Conflicts of Interest}

The authors declare that there are no conflicts of interest.

\section{Acknowledgments}

This research was funded in part by Al Maqdisi Program 2018-2020 (French Consulate Jerusalem). The authors would like to acknowledge Faculty of Health Sciences at Islamic University of Gaza, Gaza Strip, Palestine, and Faculty of Medicine and Health Sciences at An-Najah National University, West Bank, Palestine, for facilitating the accomplishment of the current study. The authors are also thankful for the Palestinian Ministry of Health.

\section{References}

[1] M. Newton-Foot, Y. Snyman, M. R. B. Maloba, and A. C. Whitelaw, "Plasmid-mediated mcr-1 colistin resistance in Escherichia coli and Klebsiella spp. clinical isolates from the Western Cape region of South Africa," Antimicrobial Resistance \& Infection Control, vol. 6, no. 1, p. 78, 2017.

[2] C. Zhu, E. K. Schneider, J. Wang et al., "Traceless reversible polymeric colistin prodrug to combat multidrug-resistant (MDR) gram-negative bacteria," Journal of Controlled Release, vol. 259, pp. 83-91, 2017.

[3] T. Sato, T. Shiraishi, Y. Hiyama et al., "Contribution of novel amino acid alterations in PmrA or PmrB to colistin resistance in mcr-negative Escherichia coli clinical isolates, including major multidrug-resistant lineages O25b: H4-ST131-H30Rx and non-x," Antimicrobial Agents and Chemotherapy, vol. 62, no. 9, pp. e00864-00818, 2018.

[4] S. Mangal, H. Park, L. Zeng et al., "particle formulations of colistin and meropenem with improved in-vitro bacterial killing and aerosolization for inhalation," International Journal of Pharmaceutics, vol. 548, no. 1, pp. 443-453, 2018.

[5] S. Kim, J. H. Woo, N. Kim et al., "Characterization of chromosome-mediated colistin resistance in Escherichia coli isolates from livestock in korea," Infection and Drug Resistance, vol. 12, pp. 3291-3299, 2014.

[6] A. Z. Bialvaei and H. Samadi Kafil, "Colistin, mechanisms and prevalence of resistance," Current Medical Research and Opinion, vol. 31, no. 4, pp. 707-721, 2015.

[7] M. Li, T. Giani, M. Raffone et al., "Colistin resistance superimposed to endemic carbapenem-resistant Klebsiella pneumoniae: a rapidly evolving problem in Italy," Eurosurveillance, vol. 19, no. 42, Article ID 20939, 2014.

[8] CLSI, Performance Standards for Antimicrobial Susceptibility Testing, Clinical and Laboratory Standards Institute (CLSI), Wayne, PA, USA, 2018.

[9] European Committee on Antimicrobial Susceptibility Testing, Breakpoint Tables for Interpretation of MICs and Zone Diameters, European Society of Clinical Microbiology and Infectious Diseases Basel, Munich, Germany, 2015.

[10] S. Harbarth, P. Rohner, E. Safran, J. Garbino, R. Auckenthaler, and D. Pittet, "Resistance to amikacin and gentamicin among Gram-negative bloodstream isolates in a university hospital between 1989 and 1994," Clinical Microbiology and Infection, vol. 4, no. 4, pp. 199-204, 1998.

[11] G. Tayh, N. Al Laham, H. Ben Yahia, R. Ben Sallem, A. E. Elottol, and K. Ben Slama, "Extended-spectrum $\beta$-lactamases among Enterobacteriaceae isolated from urinary tract infections in Gaza strip, Palestine," BioMed research international, vol. 2, 2019.

[12] M. A. Shin and M. Blok, "Multidrug resistance among Enterobacteriaceae is strongly associated with the presence of integrons and is independent of species or isolate origin," The Journal of Infectious Diseases, vol. 187, no. 2, pp. 251-259, 2003.

[13] A. A. Elmanama, M. R. Al-Reefi, E. M. Diab, and N. K. AlTawil, "Colistin resistant Escherichia coli isolated from chicken fecal materials in Gaza strip," Journal of Al Azhar UniversityGaza (Natural Sciences), vol. 20, no. 2, pp. 25-38, 2018.

[14] L. Bardet, L. Okdah, S. Le Page, S. A. Baron, and J.-M. Rolain, "Comparative evaluation of the UMIC Colistine kit to assess MIC of colistin of gram-negative rods," BMC Microbiology, vol. 19, no. 1, p. 60, 2019.

[15] W. Alfouzan, R. Dhar, and D. Nicolau, "In vitro activity of newer and conventional antimicrobial agents, including fosfomycin and colistin, against selected Gram-negative bacilli in Kuwait," Pathogens, vol. 7, no. 3, p. 75, 2018.

[16] Y. Doi, "Treatment options for carbapenem-resistant gramnegative bacterial infections," Clinical Infectious Diseases, vol. 69, no. 7, pp. S565-S575, 2019.

[17] D. M. Livermore, M. Warner, S. Mushtaq, M. Doumith, J. Zhang, and N. Woodford, "What remains against carbapenem-resistant Enterobacteriaceae? Evaluation of chloramphenicol, ciprofloxacin, colistin, fosfomycin, minocycline, nitrofurantoin, temocillin and tigecycline," International Journal of Antimicrobial Agents, vol. 37, no. 5, pp. 415-419, 2011.

[18] M. M. Zafer, H. A. El-Mahallawy, A. Abdulhak, M. A. Amin, M. H. Al-Agamy, and H. H. Radwan, "Emergence of colistin resistance in multidrug-resistant Klebsiella pneumoniae and Escherichia coli strains isolated from cancer patients," Annals of Clinical Microbiology and Antimicrobials, vol. 18, no. 1, p. 40, 2019.

[19] R. H. Rida, N. A. Al Laham, and A. A. Elmanama, "Carbapenem resistance among clinical and environmental Gramnegative isolates recovered from hospitals in Gaza strip, Palestine," Germs, vol. 8, no. 3, pp. 147-154, 2018. 